\title{
State Variable Modeling of the Power Pin Diode Using an Explicit Approximation of Semiconductor Device Equations: A Novel Approach
}

\author{
H. Morel, S. H. Gamal, and J. P. Chante
}

\begin{abstract}
The concepts of state variable modeling have been applied to obtain a general circuit like model for the power PIN diode. The main aim of this paper is to demonstrate the feasibility of the state variable modeling approach for the PIN diode. From simplified semiconductor device differential equations, the model is built with the corresponding variational equation using an internal approximation. With a special choice of the decomposition functional basis of such internal approximation, it was possible to get efficient and reliable models for the reverse recovery. A simple model of three state variables that has only six parameters, most of which are technological, represented a major improvement in describing circuit/device waveforms during reverse recovery.
\end{abstract}

\section{INTRODUCTION}

$\mathbf{M}$ ODELING of an electronic device to be used in a circuit simulator is usually based on an equivalent circuit approach. Equivalent circuits are convenient representations as they allow the description of complex systems by assembling elementary components. As an example, to model a device by the commonly used circuit simulator SPICE [1], the following steps are taken. First, the steady state equivalent circuit for forward bias conditions is found. Next, the model under reverse bias conditions is sought by adding some new components and finally the transient model is obtained by new component addition. Actually, the main problem of this method is that adding a new component can disturb the previously established model and obviously such a completely empirical technique might be difficult for many interesting cases, as for example in the case of a PIN power diode. A micro-model approach [2] might give satisfactory forward recovery circuit models for a junction diode, but the more difficult case of the reverse recovery in a complex external circuit is still laking. We believe that it is not possible, to build an efficient connected equivalent circuit model for the PIN diode, because of different natures of energy storage in the device as space charge regions store electrostatic energy, whereas carrier diffusion regions store the internal energy of electron and hole gases. Another basic approach is to solve directly the basic semiconductor equations [3] which are the Poisson's equation and the continuity equations for holes and electrons together with the external circuit equations using numerical techniques. Unfortunately, for the transient

Manuscript received January 21, 1992: revised August 2, 1993. This work was supported by the European Project PROMETHEUS.

The authors are with CEGELY, Centre de Génie Electrique de Lyon, INSA de Lyon, Bàt. 401, 69621 Villeurbanne Cedex, France.

IEEE Log Number 9214837. problems, this approach costs large CPU time and it is not very practical even for the simplest circuit configurations.

Recently a state variable modeling [4] for the PIN diode has been proposed [5], [17] as a more general alternative to the commonly used connected circuit modeling. In the present paper, we demonstrate the feasibility of such an approach. The procedure required to build the state variable model from the simplified semiconductor device equations for the more difficult case of reverse recovery is presented in detail. This procedure is based on an explicit approximation obtained from an Internal Approximation. We adapt a particular Internal Approximation modeling method, the EigenValue Internal Approximation Modeling (EVIAM), that results in a state variable model with only few state variables while keeping the model within an acceptable accuracy. A regional approximation is used where the diode is partitioned into two main regions which control the reverse recovery behavior. The two regions are the space charge region of the $P^{+} N$ junction and the carrier diffusion region which is the neutral base region. For each region a state variable model is developed where the external variables are the total current that passes through the region and the voltage drop across it. The global model is the assembly of those different local models. In the following section, the general form of the state variable model for the carrier diffusion region is derived. Then in Section III, the model of the carrier diffusion region is completely defined using the EVIAM method. The behavior of the space charge region both in the forward and reverse bias is modeled in Section IV.

\section{INTERNAL APPROXIMATION MODELING OF THE NEUTRAL Carrier Diffusion Region of A PIN Diode}

In this section, the general form of the state variable model for the carrier diffusion region will be developed. Consider a PIN diode for power electronic applications. Assuming high level injection during all the transient phases, position independent carrier lifetime and mobilities and neglected recombination in the end regions, the hole concentration is given by the classical one dimensional diffusion equation [6]:

$$
\frac{\partial p}{\partial t}(x, t)=D \frac{\partial^{2} p}{\partial x^{2}}(x, t)-\frac{p(x, t)}{\tau}
$$

with the boundary conditions

$$
\frac{\partial p}{\partial x}(0, t)=-\eta i(t)
$$




$$
\frac{\partial p}{\partial x}(w, t)=\kappa i(t)
$$

where $p(x, t)$ is the hole concentration, $\tau$ is the carrier lifetime, $D=\frac{2 \mu_{n} \mu_{p}}{\mu_{n}+\mu_{p}} U_{T}$ is the ambipolar diffusion coefficient, $\mu_{n}$ and $\mu_{p}$ are the electron and hole mobilities, respectively, $U_{T}$ is the thermal voltage, $i(t)$ is the total current that flows through this region, $\eta=\frac{1}{2 q \mu_{p} U_{T} A}, \kappa=\frac{1}{2 q \mu_{n} U_{T} A}, A$ is the diode effective area, $q$ is the elementary charge and $w$ is the width of the neutral region where the origin of the $x$ axis is taken at the edge of the neutral region. We do not consider very fast transient phenomena like those encoutered in ESD protection or microwave applications and therefore the neutrality assumption is justifed leading to no current variation in position i.e. the current $i(t)$ has the same value at the boundaries of the neutral region as given by (lb) and (1c). Equations (1a)-(1c) with the above assumptions have been widely used to determine analytically the diode initial reverse recovery when the external circuit interaction may be neglected [7]-[8], i.e. when the diode voltage drop is negligible. A solution $p(x, 0)$ of the steady state problem must be added to the above set of equations as an initial condition. The boundary conditions (1b) and (1c) are direct result of the assumption of neglected recombination in the end regions [6]-[8]. In later recovery phases, the space charge region extends and the assumption of high level injection in the neutral diffusion region may no longer be valid according to the initial free carrier concentration and carrier lifetime. However, the behavior of fast diodes during these phases is mainly governed by the space charge behavior [6].

First we obtain the variational equation [9], [10] of (1) by multiplying (la) by a time independent arbitrary trial function $\mathrm{s}(\mathrm{x})$ and integrating all the terms from 0 to $w$

$$
\frac{d}{d t} \int_{0}^{w} p s d x=\int_{0}^{w} D \frac{\partial^{2} p}{\partial x^{2}} s d x-\int_{0}^{w} \frac{1}{\tau} p s d x
$$

Integrating by parts and applying the boundary conditions ( $1 \mathrm{~b})$ and (1c) yields

$$
\begin{aligned}
\frac{d}{d t} \int_{0}^{w} p s d x=- & \int_{0}^{w} D \frac{\partial p}{\partial x} \frac{\partial s}{\partial x} d x-\int_{0}^{w} \frac{1}{\tau} p s d x \\
& +D(\eta s(0)+\kappa s(w)) i
\end{aligned}
$$

Equation (3) has the general form [10]

$$
p \in \mathbf{V}, \frac{d}{d t} m(p, s)+g(p, s)=\ell(s, i), \forall s \in \mathbf{V}
$$

where

$$
\begin{aligned}
m(p, s) & =\int_{0}^{w} p s d x \\
g(p, s) & =\int_{0}^{w} D \frac{\partial p}{\partial x} \frac{\partial s}{\partial x} d x+\int_{0}^{w} \frac{1}{\tau} p s d x
\end{aligned}
$$

and

$$
\ell(s, i)=D(\eta s(0)+\kappa s(w)) i
$$

where $\mathbf{V}$ is a functional space that contains the solution $p$. The variational equation (4) is the weak form [10] of the boundary value problem defined by (1) and which allows Functional Analysis developments. In general, it is not possible to obtain an exact solution for (4). Therefore, we seek an approximate solution $p_{n}(x, t) \in \mathbf{V n}$, for the carrier concentration $p(x, t) \in \mathbf{V}$, where $\mathbf{V n}$ is a functional space of finite dimension and which is included in the space $\mathbf{V}$. This method is called Internal Approximation. In fact, Internal Approximation allows to transform a boundary value problem, constituted of partial differential equations in space and time, into a finite set of differential equations in time only by the use of an explicit approximation. The method is based on choosing a suitable decomposition functional basis such that

$$
p_{n}(x, t)=\sum_{1}^{n} \xi_{k}(t) w_{k}(x)
$$

where $w_{k}, k=1, n$ is a basis of the space $\mathbf{V n}$ and $\xi_{k}(t)$ are the coordinates in this basis. The internal approximation of $p$ is the function $p_{n}$ which is the element of the space $V n$ which satisfies the following variational equation:

$$
p_{n} \in \mathbf{V}_{n}, \frac{d}{d t} m\left(p_{n}, s\right)+g\left(p_{n}, s\right)=\ell(s, i), \forall s \in \mathbf{T}_{n}
$$

where $\mathbf{T} n \subset \mathbf{V}$ is the trial functional space of the same dimension as $V n$ and which can be described by the basis $s_{j}, j=1, n$. Writing (9) for the basis functions $s_{j}$ and then combining with (8) taking into account the bilinear property of $m$ and $g$ and the time independence of $w_{k}$ and $s_{j}$ we get the following matrix equation:

$$
\mathbf{M} \frac{d \xi}{d t}+\mathbf{G} \xi=\mathbf{L}(i(t))
$$

where

$$
\begin{aligned}
M_{k j} & =m\left(w_{j}, s_{k}\right), \quad G_{k j}=g\left(w_{j}, s_{k}\right), \\
L_{j}(i) & =\ell\left(s_{j}, i\right), \quad k, j=1, n
\end{aligned}
$$

and $\xi^{T}=\left[\xi_{1}, \ldots, \xi_{n}\right]$ is a vector of order $n, \mathbf{L}$ is a vector which is a function of the external total current. Once the basis functions $w_{k}$ and the trial functions $s_{j}$ are specified, the coefficients of the matrices $\mathbf{M}, \mathbf{G}$ and $\mathbf{L}$ can be obtained using (5), (6) and (7), respectively. For the case of the finite element method [3][9], the basis functions are defined such that they vanish everywhere except over a small interval element and the trial functions are the same as the basis functions, i.e. $\mathbf{T} n$ is the same space $\mathbf{V} n$. In the next section, however, we apply another method, the EVIAM method, which is more suitable to our purpose, that is to build a state variable model with a few state variables while keeping the model within an acceptable accuracy. The interest in a state variable model arises from the fact that in practice it is interesting to integrate a device model into a circuit simulator, that considers the concepts of state variable modeling, to obtain the behavior of a complete system. Finally (10) can be written as

$$
\frac{d \mathbf{x}}{d t}=-\mathbf{D} \mathbf{x}+\mathbf{L}(i)
$$

where

$$
\mathrm{D}=\mathrm{GM}^{-1}
$$


and the state vector $\mathbf{x}$ is given by

$$
\mathbf{x}=\mathbf{M} \xi
$$

In deriving (12), moving boundary effects, that arise when the space charge region extends, have not been considered. It is theoretically possible to take into account those effects in the variational equation (4). For reasons of simplifying the model, such effects are rather taken into account using the empirical expression (26) as dissussed in Section IV.

To complete this local state model, a relation for the second external variable which is the voltage drop across the diffusion region $u_{d}$ is required. This is directly given by [11]

$$
u_{d}=R_{d} i
$$

where

$$
R_{d}=\frac{1}{A} \int_{0}^{w} \frac{d x}{q\left(\mu_{n}+\mu_{p}\right) p(x)}
$$

The Dember voltage component [11] that arises from the difference between the carrier concentrations $p(0)$ and $p(w)$ is neglected in (15), as it is practically small. The resistance $R_{d}$ can be calculated from (14) and (16) using the approximation $p_{n}$ defined by (8) and therefore, it can be considered as a function of the state vector $\mathbf{x}$. Then equation (15) can be rewritten as

$$
0=R_{d}(\mathbf{x}) i-u_{d}
$$

Clearly (12) and (17) have the general state variable form [4][5]

$$
\begin{aligned}
\frac{d \mathbf{x}}{d t} & =\mathbf{f}(\mathbf{x}, \mathbf{y}) \\
0 & =\mathbf{b}(\mathbf{x}, \mathbf{y})
\end{aligned}
$$

where $\mathbf{y}=\left(\begin{array}{c}i \\ u_{d}\end{array}\right)$ is the external variable vector, $\mathbf{f}$ is the vectorial internal function and $\mathbf{b}$ is the vectorial bond function.

\section{Elaboration of the STate Variable MODEL FOR THE DIFFUSION REGION}

In the previous section, we developed the state variable model of the carrier-diffusion region from the variational equation using the concepts of Internal Approximation. To calculate the involved matrices $\mathbf{M}, \mathbf{G}$ and $\mathbf{L}$, the decomposition functions $w_{k}$ and the trial functions $s_{j}$ must be specified. Although theoretically the choice of $w_{k}$ and $s_{j}$ is arbitrary, some choices are more efficient regarding the accuracy of the internal approximation for a given number of functions. Our criterion of choice is given in what follows. First we write (1a) in the following operator form

$$
\text { Diff } p=\frac{\partial p}{\partial t}
$$

where the operator Diff is defined by

$$
\operatorname{Diff}=D \frac{\partial^{2}}{\partial x^{2}}-\frac{1}{\tau}
$$

A necessary condition that the internal approximation (8) satisfies exactly the boundary conditions (1b) and (1c) is that the decomposition functions $w_{k}$ satisfy the following condition

$$
-\frac{1}{\eta} \frac{d w_{k}}{d x}(x=0)=\frac{1}{\kappa} \frac{d w_{k}}{d x}(x=w)
$$

Also it should be noted (as demonstrated in Appendix B) that a sufficient condition for the internal approximation (8) to be the exact solution of equation set (1a)-(1c) in steady state is that $w_{1}$ is chosen such that

$$
\left\{\begin{array}{c}
\text { Diff } w_{1}=0 \\
-\frac{1}{\eta} \frac{d w_{1}}{d x}(x=0)=\frac{1}{\kappa} \frac{d w_{1}}{d x}(x=w) \\
\frac{1}{\kappa} \frac{d w_{1}}{d x}(x=w)>0
\end{array}\right.
$$

i.e. $w_{1}$ is an eigenfunction of Diff corresponding to the zero eigenvalue. Our criterion is to apply the EVIAM (EigenValue Internal Approximation Modeling) method for which the decomposition functions $w_{k}$ and the trial functions $s_{j}$ are some eigenfunctions of the operator Diff that satisfy (21) and (22). In Appendix A these eigenfunctions have been studied in detail. In general we take the hyperbolic eigenfunctions $r_{k}(x)$ as decomposition basis functions $w_{k}(x)$ and the trigonometric eigenfunctions $q_{j-1}(x)$ as trial functions $s_{j}(x)$. Notice that $r_{1}(x)$ satisfies condition (22) (as derived in Appendix A) and consequently our internal approximation is an exact solution for the steady state problem. The choice of the trigonometric eigenfunctions $q_{j-1}$ as trial functions simplifies the calculation of the matrices $\mathbf{M}, \mathbf{G}$ and $\mathbf{L}$. Moreover, with the particular eigenfunction sequences defined by (A7) and (A8), the matrix D given by (13) becomes diagonal with the diagonal elements given by

$$
D_{k k}=\frac{1}{\tau}\left(1+(k-1) \frac{\pi^{2} D \tau}{w^{2}}\right), \quad k=1,2, \ldots
$$

The vector $\mathrm{L}$ can be calculated using (11) and (7). The elements of this vector $L_{j}$ are given by

$$
L_{j}(i)=\left\{\begin{array}{cc}
\frac{i}{\tau I_{o}} & \text { for } j \text { odd } \\
\frac{i}{\tau I_{o}} \frac{\mu_{n}-\mu_{p}}{\mu_{n}+\mu_{p}} & \text { for } j \text { even }
\end{array}\right.
$$

where $I_{o}$ is a reference current. The simplest model is built using only two eigenfunctions for each basis resulting in a two state variable model. In Section V, we will show that such model, built only with the hyperbolic eigenfunctions $r_{1}, r_{2}$ and the trigonometric eigenfunctions $q_{0}, q_{1}$, has a very small calculation time with a good accuracy. In this case, with some manipulation, (12) is explicitly given by

$$
\begin{aligned}
\frac{d}{d t}\left(\begin{array}{l}
x_{1} \\
x_{2}
\end{array}\right)=- & \frac{1}{\tau}\left(\begin{array}{cc}
1 & 0 \\
0 & 1+\frac{\pi^{2} D \tau}{w^{2}}
\end{array}\right)\left(\begin{array}{l}
x_{1} \\
x_{2}
\end{array}\right) \\
& +\frac{i}{\tau I_{o}}\left(\begin{array}{c}
1 \\
\frac{\mu_{n}-\mu_{p}}{\mu_{n}+\mu_{p}}
\end{array}\right)
\end{aligned}
$$




\section{The State Variable Model of THE SPACE CHARGE REgION}

In this section, the state variable model for forward and reverse bias of the $p$ - $n$ space charge region will be introduced. As a simple charge control model is sufficient for the depleted junction case we do not need to apply the EVIAM method. The electron current $i_{n}$ at the $n$-side edge of the space charge region is no longer equal to zero because it arises in fact from the electrons extracted while the space charge region extends to support the reverse bias, i.e.

$$
i_{n}=-\frac{d}{d t}\left(q N_{D} A w_{c}\right)
$$

where $w_{c}$ is the extension of the space charge region in the $n$-side and $N_{D}$ is the uniform base doping concentration. The positive sense of the current $i_{n}$ in (26) is from the $p$-side towards the $n$-side when $w_{c}$ increases. Therefore, the total current $i$ is given by:

$$
i=i_{n}+i_{p}
$$

Combining (26) and (27) we get

$$
\frac{d w_{c}}{d t}=\frac{i_{p}-i}{q N_{D} A}
$$

Equation (28) defines the state variable model of the reverse biased space charge region where $w_{c}$ is the only state variable in this local model and the total current $i$ is the first external variable. The second external variable is the reverse voltage drop $u_{c}$ on this region which is related to $w_{c}$ in a one-sided junction model [12] by

$$
\phi-u_{c}=\frac{U_{T}}{2}\left(\frac{w_{c}}{L_{\mathrm{ND}}}\right)^{2}
$$

where $\phi$ is the junction built-in voltage and $L_{\mathrm{ND}}=\sqrt{\frac{\varepsilon U_{T}}{q N_{D}}}$ is the Debye length in the $n$-side. An empirical expression for $i_{p}$ is suggested such that

$$
\frac{i_{p}}{I_{o}}=-x_{1} \frac{\tau}{\tau_{D}}\left(1+\alpha \frac{w_{c}}{L_{\mathrm{ND}}}\right)
$$

where $\tau_{D}$ and $\alpha$ are two adjustable parameters. $\tau_{D}$ corresponds to a proportional factor between $i_{p}$ ans $x_{1}$ which accounts for the effects of the stored charge in the diffusion region. The parameter $\alpha$ corresponds to the effects of moving diffusion region boundaries that arise when the space charge region width $w_{c}$ changes with time. Thus when the space charge region is reverse biased, the role of the carrier diffusion region in the recovery behavior is taken into account by considering the state variable $x_{1}$ in (30).

When the space charge region is forward biased $w_{c}$ is always very small and there is no more inertial effect as in the case of reverse bias. This means that we do not need any state equation. However, the continuity of the state variable $w_{c}$ has to be preserved [4]. For this purpose, a relation between $w_{c}$ and the carrier density $p_{1}$ at the edge of the space charge region in the n-side can be used,

$$
w_{c}=\sqrt{\frac{2 \varepsilon U_{T}}{q p_{1}}}
$$

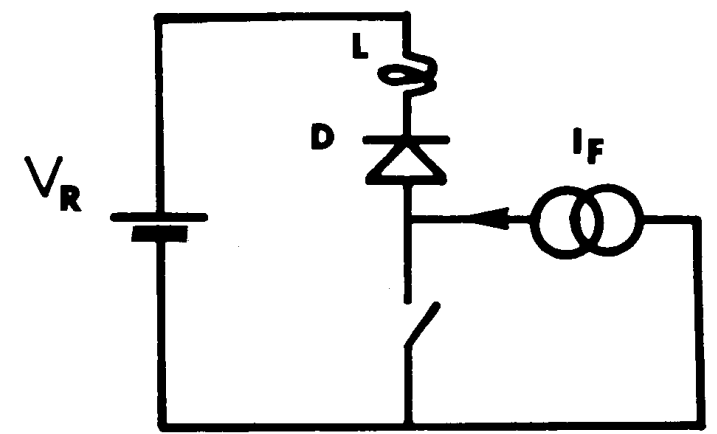

Fig. 1. Ramp recovery circuit of PIN diode.

The complete derivation of (31) is given in Appendix C. The hole density $p_{1}$ is calculated from (8) at $x=0$. This means that $w_{c}$ depends on the values of the state variables in the neutral diffusion region. Finally, the voltage drop $u_{c}$ on the space charge region can be calculated using (29). A more accurate relation which takes into account the effect of high level injection in the n-side on the width of the space charge region is derived in Appendix C. This relation reads

$$
\Phi-u_{c}=U_{T} \ln \left(\frac{1}{2}\left(\frac{w_{c}}{L_{\mathrm{ND}}}\right)^{2}\right)+U_{T} \ln \left(\frac{N_{A}}{N_{D}}\right)
$$

\section{RESULTS AND DisCUSSIONS}

The global state model that we have established in the previous sections and which is described by (25) and (28) and the corresponding bond equations is based on the three state variables $x_{1}, x_{2}$ and the width of the space charge region $w_{c}$. The parameters of this model are the base width $w$, the base doping concentration $N_{D}$, the carrier lifetime $\tau$, the effective area $A$ and two adjustable parameters $\alpha$ and $\tau_{D}$. As an illustrating exemple, the model is used to predict the behavior of ramp recovery [13] where the diode was initially in forward conduction and then reverse biased by a voltage source $V_{R}$ in series with an inductance $L$ (Fig. 1). The rate of the current ramp di/dt in this case is approximately equal to $V_{R} / L$. This is in fact the simplest circuit configuration commonly encountered in practice. The current and the voltage have been observed using a Tektronix digital storage oscilloscope model 2432 and 6137 voltage and 6022 current Tektronix probes. The circuit simulator PACTE [14] that we developed to fit well with the state variable concepts is used. In this simulator the model equations and the external circuit equations are solved simultaneously by the Gear method [15] to obtain the recovery behavior. The model parameters can be determined by minimizing the differences between simulation and experimental switching parameters for a given switching condition. A first estimate for some parameters can be also made using the manufacturer's specifications. For example, the area $\mathrm{A}$ can be estimated from the nominal current, and the base width $\mathrm{w}$ and doping concentration $N_{D}$ can be estimated from the breakdown voltage. 
Fig. 2 shows the current and voltage waveforms obtained for a BYT 12 PI 600 diode (The breakdown voltage $V_{B R}$ is of $600 \mathrm{~V}$ ) for a forward current of $0.63 \mathrm{~A}$ and a reverse applied voltage of $80 \mathrm{~V}$. The resulting current rate was $41 \mathrm{~A} / \mu \mathrm{s}$, which corresponds to an inductance of $1.9 \mu \mathrm{H}$. The corresponding evolution of the carrier distribution in the diffusion region is shown in Fig. 3. For the diode tested, the following parameters were obtained: carrier lifetime $\tau=130 \mathrm{~ns}$, effective area $A=$ $0.12 \mathrm{~cm}^{2}$, base width $w=40 \mu \mathrm{m}$, base doping concentration $N_{D}=2 \times 10^{14} \mathrm{~cm}^{-3}$. The parameters $\alpha$ and $\tau_{D}$ are 0.045 and $0.8 \mathrm{~ns}$, repectively. They are found to vary around those values from one diode to diode and they affect only the softness of the recovery as they intervene only when the space charge region is reverse biased, i.e. when the reverse voltage starts to increase across the diode. As shown in Fig. 2, a good agreement is obtained between simulation and experimental waveforms for both the current and voltage during the most of the recovery time. Moreover, the calculation time was only $57 \mathrm{~s}$ on a $80286 \mathrm{PC}$. For these results the voltage drop across the diffusion region $u_{d}$ which is given by (15) and (16) was neglected to simplify the calculation. A damping effect was thus neglected which resulted in the slowly damping behavior at the end of the recovery as shown in Fig. 2.

From the point of view of power circuit designer, some switching parameters are very important such as the maximum reverse current $I_{\mathrm{RM}}$, the recovery time between the first two successive zero current crossing $t_{\mathrm{rr}}$ and the maximum voltage overshoot $V_{\mathrm{RM}}$. The first two parameters affect the turn-off power losses and the third indicates the reliability of the diode under certain switching conditions. Measured and calculated switching parameters for the same tested diode are given in Table I for three different switching cases defined in Table II. A satisfactory agreement is generally obtained as demonstrated by Table I. It should be also noted that those results are obtained using the simplest model which is based on only two state variables $x_{1}$ and $x_{2}$ for the carrier diffusion region. There are also some simplifications in the physical model such as position independent carrier lifetime and mobilities in the diffusion region and neglected recombination in the end regions. However, those approximations are only of secondary importance [13].

The same model has been also successfully used to predict the diode reverse recovery in many other more sophisticated but interesting cases like chopper and invertor circuits [14] or high frequency resonant converters [16].

The fact that the calculation time is short is mainly due to the internal approximation method used to build the state variable model. With the EVIAM method it was possible to obtain a model that has only few state variables while keeping the model within an acceptable accuracy. As it has been mentioned in Section III, the model based on (25) is obtained using the hyperbolic eigenfunctions $r_{1}(x)$ and $r_{2}(x)$ which are defined by (A3) and (A4). Those functions, which serve as decomposition basis functions, are illustrated in Fig. 4. For a given instant of time the carrier concentration is a linear composition of those two functions. For instance, at $t=t_{0}$ in Fig. 3, which corresponds to the steady state solution, the carrier distribution is composed only of $r_{1}$. If we

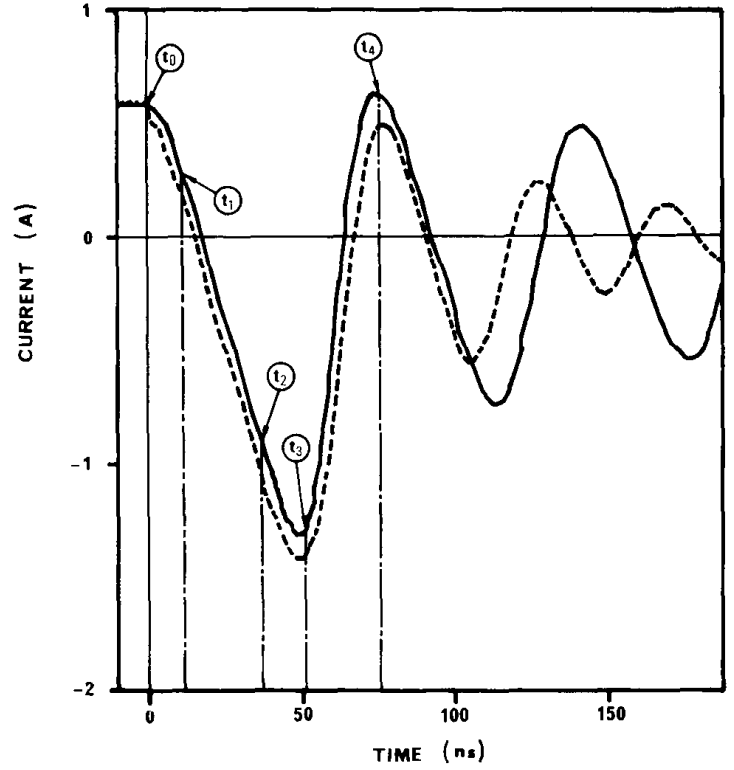

(a)

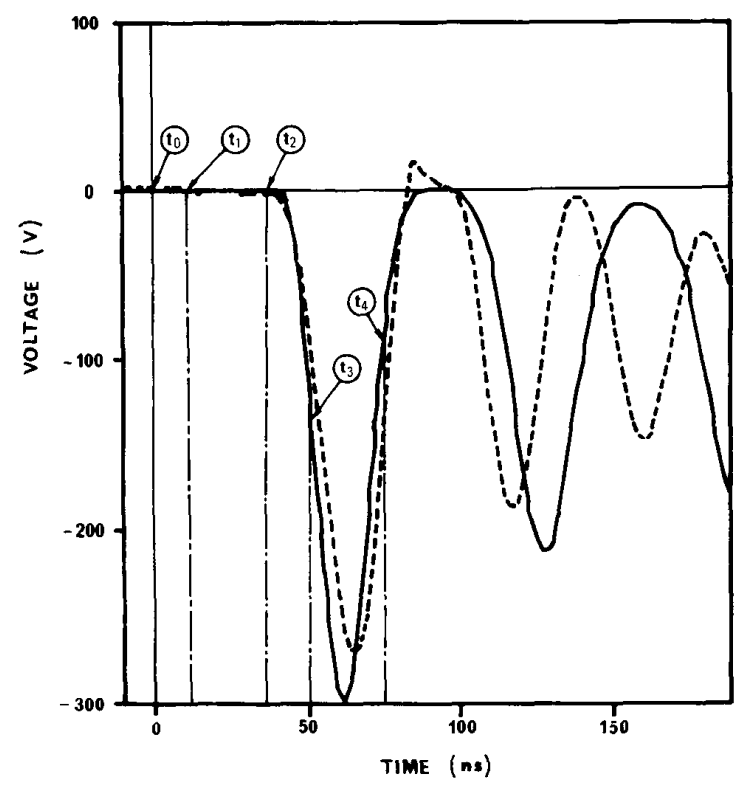

(b)

Fig. 2. Simulated (solid lines) and experimental (dashed lines) transient waveforms in ramp recovery for a BYT $12 \mathrm{Pl} 600$ diode. (a) Current waveforms. (b) Voltage waveforms. The forward current $I_{F}=0.63 \mathrm{~A}$ and the reverse applied voltage $V_{R}=80 \mathrm{~V}$. The inductance $L$ accounts to $1.9 \mu \mathrm{H}$.

apply the finite element method [3][9] instead of the EVIAM method to define the basis functions and the trial functions we must use a large number of basis functions to obtain a good approximation. This can be easily illustrated using Fig. 5 , where, as an illustrating example, the carrier distribution given in Fig. 3 at $t=t_{2}$, is reproduced. A finite element solution that uses 11 linear basis functions ( 10 elements are required in this case) might produce the carrier distribution shown in Fig. 5. with the same order of accuracy as shown 


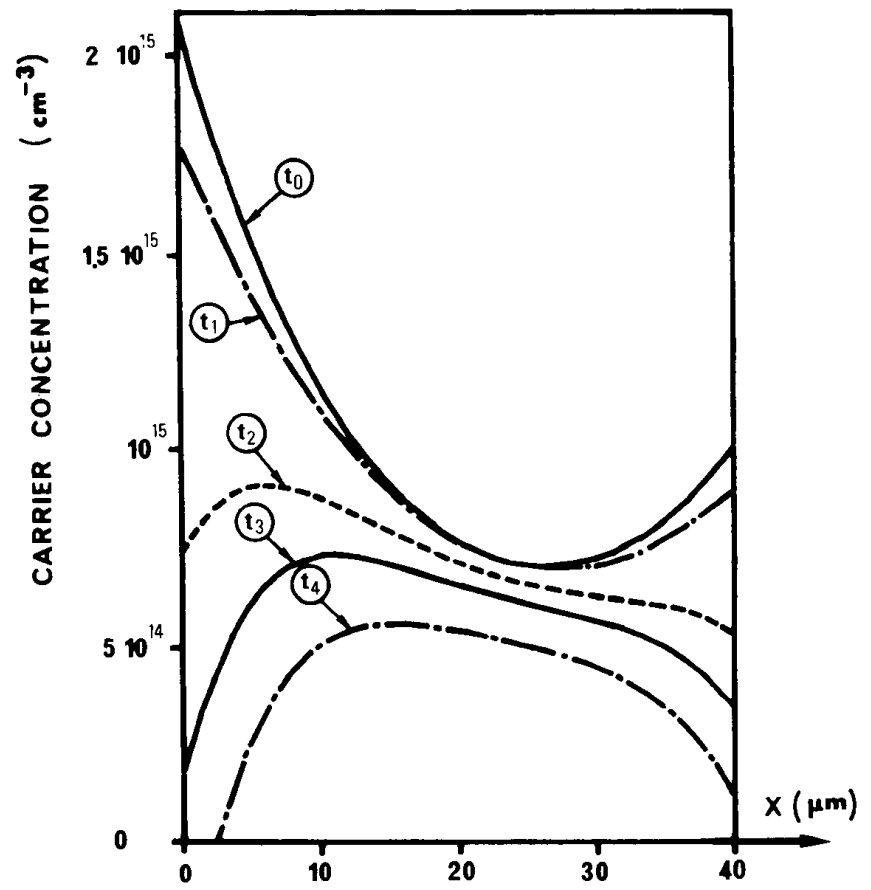

Fig. 3. Evolution of carrier distribution in the diffusion region during the reverse recovery of current and voltage waveforms shown in Fig. 2 . $t_{0}=0$ $\mathrm{ns}, t_{1}=12.0 \mathrm{~ns}, t_{2}=36.84 \mathrm{~ns}, t_{3}=51.10 \mathrm{~ns}$ and $t_{4}=76.0 \mathrm{~ns}$.

by the piecewise linear approximation. It is clear that less functions would produce a worse approximation.

It should be noted that the methods described above are not only confined to the diffusion region of the power diode. They can be also applied to any region where there exist distributed storage effects as in bipolar transistor, thyristor and other power devices.

\section{CONCLUSION}

A new approach for predicting semiconductor device behavior in reverse recovery is applied for the power PIN diode. This approach leads to efficient state variable models that can account for the device/circuit interaction during reverse recovery. The state variable model is derived using the internal approximation of the variational problem. We used the EVIAM method which specifies the basis functions and the trial functions from the eigenfunctions of the diffusion operator Diff to build the model. An efficient model of only three state variables and six parameters has been obtained. The model parameters are easy to determine and most of them are technological. Moreover the calculation time is very small. So we can see that the model exhibits considerable improvements over conventional equivalent circuit models of power diodes.

APPENDIX A

THE EINGENFUnCTIONS OF THE OPERATOR DIFF

Studying the differential equation defined by

$$
\text { Diff } p(x)=\lambda p(x)
$$

where the operator Diff is defined by (20). Its characteristic equation

$$
r^{2}-\left(\frac{\lambda}{\mathbf{D}}+\frac{1}{\mathbf{D} \tau}\right)=0
$$

have three cases:

(i) if $\lambda>-1 / \tau$ there are two independent hyperbolic solutions,

(ii) if $\lambda<-1 / \tau$ there are two independent trigonometric solutions and

(iii) if $\lambda=-1 / \tau$ there are two independent critical solutions.

We consider the first two cases briefly. For the hyperbolic solution where $\lambda$ is greater than $-1 / \tau$, we can define $\lambda$ such that

$$
\lambda_{h}=\frac{-1}{\tau}+\frac{D}{h^{2}}
$$

where $h$ is a real number. In this case, the general solution of (A1) is

$$
r(x)=A \cosh \left(\frac{x-\phi}{\mathbf{h}}\right)
$$

so the following functions are solution of (A1) and satisfy (21)

$$
r_{h}(x)=\left.h \eta\right|_{o} \frac{\cosh \left(\frac{x-\phi_{h}}{h}\right)}{\sinh \left(\frac{\phi_{h}}{h}\right)}
$$




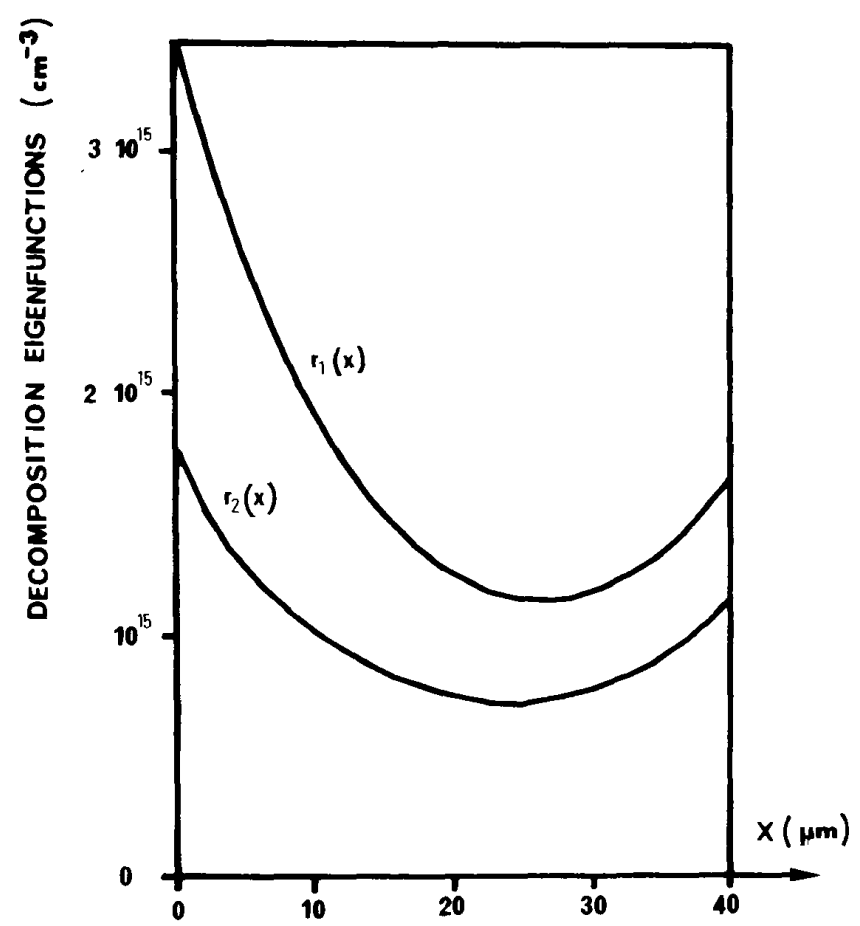

Fig. 4. The decomposition basis functions $r_{1}(x)$ and $r_{2}(x)$ as defined by (A3) and (A4). They are calculated using the model parameters of the BYT 12 Pl 600 tested diode.

where $I_{o}$ is a reference current and $\phi_{h}$ is given by

$$
\phi_{h}=h \tanh ^{-1}\left(\frac{\mu_{n} \sinh \left(\frac{w}{h}\right)}{\mu_{p}+\mu_{n} \cosh \left(\frac{w}{h}\right)}\right)
$$

For the trigonometric solution, $\lambda$ is less than $-1 / \tau$, then we can define

$$
\lambda_{h}=\frac{-1}{\tau}-\frac{D}{h^{2}}
$$

In this case, the general solution of (A1) is

$$
q(x)=A \cos \left(\frac{x-\delta}{h}\right)
$$

So the following functions are solution of (A3) and satisfy (21)

$$
q_{h}(x)=\cos \left(\frac{x-\delta_{h}}{h}\right)
$$

where $\delta_{h}$ is given by

$$
\delta_{h}=h \tan ^{-1}\left(\frac{\mu_{n} \sin \left(\frac{w}{h}\right)}{\mu_{p}+\mu_{n} \cos \left(\frac{w}{h}\right)}\right)
$$

Since functions $r_{h}$ and $q_{h}$ satisfy (A1), they are eigenfunctions of the operator Diff with the corresponding eigenvalue $\lambda_{h}$. We choose a particular eigenfunctions sequence, which might serve as a decomposition basis function sequence and which is defined by the following discrete values of the parameter $h$

$$
h_{k}=\frac{\sqrt{D \tau}}{k}, \quad k=1,2,3 \ldots
$$

With this sequence $r_{1}(x)$ satisfies (22) while the rest of the $r_{k}$ functions well approximate the solution of (1) as given for example in [7] for the initial phase of the diode recovery. Now we also define a particular eigenfunction sequence to be used for generating the trial basis functions and which yields a diagonal matrix $D$. This sequence is defined by the following discrete values of the parameter $h$ :

$$
h_{j}=\frac{w}{j \pi} \quad j=1,2,3 \ldots
$$

It is interesting to note that with the sequence (A8) $q_{0}(x)=1$. As a result (3) reduces to

$$
\frac{d}{d t} \int_{0}^{w} p_{n} d x=-\frac{1}{\tau} \int_{0}^{w} p_{n} d x+\frac{i(t)}{q A}
$$

with $q_{0}$ and $p_{n}$ subsituted for $s$ and $p$, respectively. Equation (A9) implies that the internal approximation $p_{n}$ conserves the total electron (hole) charge.

\section{APPENDIX B}

The InTERnal Approximation in Steady State

In steady state we have from (10)

$$
\mathbf{G} \xi=\mathbf{L}(l)
$$

where $l$ is the steady state current. We will show that (22) is a sufficient condition for the internal approximation (8) to be the exact solution of equation set (1a)-(1c) in steady state: 


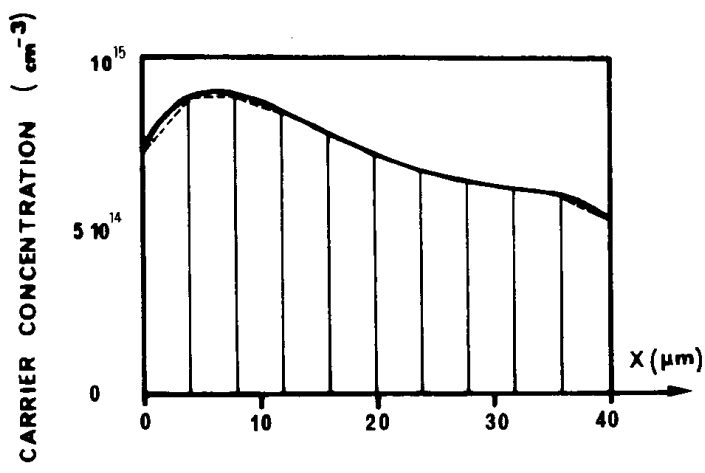

Fig. 5. Carrier distribution at $t=t_{2}$ in Fig. 2, calculated using the EVIAM method (solid lines) with only two decomposition functions and a corresponding possible linear finite element approximation (dashed lines) using eleven decomposition functions.

TABLE I

Simulated and Experimental Switching Paramaters for Three Different Switching Cases (a), (b) And (c)

\begin{tabular}{|c|c|c|c|c|c|c|}
\hline & \multicolumn{3}{|c|}{ Experiment } & \multicolumn{3}{|c|}{ Simulation } \\
\hline & (a) & (b) & (c) & (a) & (b) & (c) \\
\hline$\frac{d i}{d t}(-A / \mu s)$ & 32 & 18.5 & 41 & 30.2 & 18 & 41 \\
\hline $\mathrm{m} I_{R M}$ (A) & 1.48 & .086 & 1.4 & 1.36 & 0.83 & 1.3 \\
\hline$I_{R W}(\mathrm{~V})$ & 221 & 127 & 275 & 220 & 128 & 298 \\
\hline$t_{r} \cdot(\mathrm{ns})$ & 75 & 80 & 52 & 63 & 68 & 49 \\
\hline
\end{tabular}

Consider the vector $\sigma$ and the function $\rho(x)$ defined by

$$
\sigma_{k}=\left\{\begin{array}{cc}
-\frac{\eta l}{\frac{d w_{1}}{d x}(x=0)} \text { for } k=1, & \frac{d w_{1}}{d x}(x=0)>0 \\
0 & \text { otherwise }
\end{array}\right.
$$

and

$$
\rho(x)=\sum_{1}^{n} \sigma_{k} w_{k}(x)
$$

From (22), (B1) and (B2) we have

$$
\text { Diff } \rho=\sum_{1}^{n} \sigma_{k} \text { Diff } w_{k}(x)=0
$$

We can show that $\sigma$ is the solution of (B1): Combining (2), (3), (6) and (7) we get

$$
\int_{0}^{u^{\prime}} \operatorname{Diff} p s d x=-g(p, s)+\ell(s, l)
$$

Using $p=\rho$ and $s=s_{j}$ in (B5) and then combining with (B4) we get

$$
g\left(\rho, s_{j}\right)=\ell\left(s_{j}, l\right)
$$

From the definition of $\rho$ given by (B3) and the linear property of $g$ we get

$$
\sum_{1}^{n} \sigma_{k} g\left(w_{k}, s_{j}\right)=\ell\left(s_{j}, l\right)
$$

TABLE II

Three Different Switching Conditions (a), (b) AND (c)

\begin{tabular}{cccc} 
& (a) & (b) & (c) \\
\hline$I_{F}(\mathrm{~A})$ & 1.39 & 1.02 & 0.63 \\
$V_{R}(\mathrm{~V})$ & 61 & 36 & 80
\end{tabular}

or

$$
\mathbf{G} \sigma=\mathbf{L}(l)
$$

Then $\sigma$ is the solution of (B1) and therefore from (8) $\rho=p_{n}$, i.e.

$$
\text { Diff } p_{n}=0
$$

In addition we can easily show that $\rho$ defined by (B2) and (B3) satisfies (1b) and (1c) then $p_{n}$ is the steady state solution of (1a)-(1c).

\section{APPENDIX C}

The Space Charge Region Under High LEVEL INJECTION CONDITIONS

With the assumption of quasi-equilibrium in the space charge region, which implies constant quasi-Fermi levels for holes and electrons, the space charge region can be characterized by solving only Poisson's equation,

$$
\frac{\mathrm{dE}}{d x}=\frac{q}{\varepsilon}(p-n+N)
$$

where $E$ is the electric field and $N$ is the net doping concentration. For one-sided abrupt $p^{+} n$ junction of width $w_{c}$, the net doping concentration is the donor concentration $N_{D}$. In equilibrium and reverse bias, the common Shockley's depletion approximation is used to obtain an analytical solution where the free carrier densities $n$ and $p$ are neglected in (B1). When the neutral $n$-side is in high level injection the electron and hole densities are much greater than the donor concentration $N_{D}$ and the free carrier densities can no longer be neglected in the space charge region. Moreover, the hole density is always greater than the electron density and doping concentration. Therefore, (B1) can be reduced to

$$
\frac{\mathrm{dE}}{d x} \sim \frac{q}{\varepsilon} p(x)
$$

The hole density is given by [13]

$$
p(x)=n_{i} \exp \left(-\frac{\Phi_{p}-\Psi(x)}{U_{T}}\right)
$$

where $\Phi_{p}$ is the quasi-Fermi level for holes and $\Psi(x)$ is the electrostatic potential. At the edge $x=0$ of the space charge region at the $p^{+}$-side, we assume low level injection, hence $p(0) \sim N_{A}$. Then the constant quasi-Fermi level can be eliminated from (B3),

$$
p=N_{A} \exp \left(\frac{-\Psi(x)}{U_{T}}\right)
$$

where $\Psi(0)$ is taken as a reference equal to zero. Multiplying the two sides of (B2) by the electric field $E\left(E=-\frac{d \Psi}{d x}\right)$ and 
integrating using (B4) and assuming that $E\left(w_{c}\right)=0$, we get

$$
\frac{1}{2} \varepsilon E^{2}=q U_{T}\left[p(x)-p\left(w_{c}\right)\right]
$$

from which and taking into account the sign of the electric field, we get

$$
\frac{d \Psi}{d x}=\sqrt{\frac{2 q U_{T}}{\varepsilon}} \sqrt{p(x)-p\left(w_{c}\right)}
$$

Except for $x$ very near to $x=w_{c}$ we can fairly assume that $p(x) \gg p\left(w_{c}\right)$. With this approximation (B6) can be integrated using again (B4) to get

$$
\begin{aligned}
w_{c} & =\sqrt{2 \frac{\varepsilon U_{T}}{q N_{A}}} \exp \frac{u_{B}}{2 U_{T}} \\
& =\sqrt{2 \frac{\varepsilon U_{T}}{q p\left(w_{c}\right)}}
\end{aligned}
$$

where $u_{B}=\Psi\left(w_{c}\right)$ is the barrier height. As the barrier height is equal to the difference between the built-in voltage $\phi$ and the voltage drop on the junction $u_{c}$ we obtain (32) directly from $(\mathrm{C} 7)$

\section{REFERENCES}

[1] L. W. Nagel, "SPICE2, A computer program to simulate semiconductor circuits," Electron. Research Lab., University of California, Berkeley, MEM no. ERL-M520, 1975.

[2] Y. C. Liang and V. J. Gosbell, "Diode forward and reverse recovery model for power electronic SPICE simulations," IEEE Trans. Power Electron., vol. 5, pp. 346-356, 1990.

[3] S. Selberher, Analysis and Simulation of Semiconductor Devices. New York: Springer-Verlag/Wien, 1984

[4] D. Karnopp and R. Rosenberg, System Dynamics: A Unified Approach. New York: Wiley Interscience, 1975.

[5] O. Hamel, H. Morel, K. Besbes and J. P. Chante, "Behavioural simulation of diode devices in power systems," Proc. of the International Congress IMACS, Paris-France, vol. 3, pp. 219-223, 1988.

[6] H. Benda and E. Spenke, "Reverse recovery processes in silicon power rectifiers," in Proc. IEEE, vol. 55, pp. 1331-1354, 1967.

[7] F. Berz, "Ramp recovery in $p$-i-n diodes," Solid-State Electron., vol. 23 , pp. 783-792, 1980.

[8] F. Berz, "Step recovery of $p$-i-n diodes," Solid-State Electron., vol. 22, Pp. 927-932, 1979.

[9] G. Strang and G. J. Fix, An Analysis of the Finite Element Method. New York: Prentice Hall, 1973.

[10] R. E. Showalter, Hilbert Space Methods for Partial Differential Equation. New York: Pitman, 1979

[11] F. Berz, "A simplified theory of the $p-i-n$ diode," Solid-State Electron. vol. 20, pp. 709-714, 1977.

[12] S. M. Sze, Physics of semiconductor devices. New York: Wiley \& Sons, 1981.
[13] S. H. Gamal, H. Morel, and J. P. Chante, "Carrier lifetime measurement by ramp recovery of $p-i-n$ diodes," IEEE Trans. Electron. Devices, vol. ED-37, pp 1921-1924, 1990.

[14] H. Morel, B. Allard and J. P. Chante, "PACTE: A behavioral simulator for power electronics," in Proc. of the International Congress IMACSTCI'90, Nancy-France, pp. 511-416, 1990.

[15] L. F. Shampine and C. W. Gear, "A user's view of solving stiff ordinary differential equations," Siam Review, vol. 21, no. 1, 1979.

[16] M. Bensoam "Alimentation à découpage pseudo-resonnante: analyse et simulation du comportement á fréquence élevée," Thése de doctorat, ENSIEG, France, 1991

[17] P. O. Lauritzen and Cliff L. Ma, "A simple diode model with reverse recovery," IEEE Trans. Power Electron., vol. 6. pp. 188-191, Apr. 1991.

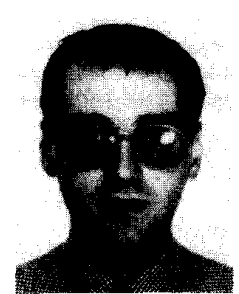

Harvé Morel was born in Reims, France in 1959. He received the engineer degree and Ph.D. in engineering from L'Ecole Centrale De Lyon, Lyon, France in 1982 and 1985, respectively.

In 1985 he joined the CNRS, Centre National de la Recherche Scientifique at CEGELY, Centre de Génie Electrique de Lyon. He manages the power semiconductor device modeling and circuit simulation team.

Dr. Morel develops the system simulator PACTE which is based on bond graph and Petri Net technics.

Salah Hassan Gamal was born in Cairo, Egypt, in 1958. He received the B. Sc. and M. Sc. degrees in electrical engineering from Ain Shams University, Cairo, in 1981 and 1985 , respectively.

He received the Ph.D. degree from the INSA (Institut Nartional des Sciences Appliquées), Lyon, France in 1992.

In 1988 he joined CEGELY, Lyon, as research assistant. Since 1992 he has been Assistant Professor in the Department of Engineering, Physics and Mathematics, Faculty of Engineering, Ain Shams University, where his research activities are devoted to power device modeling and high-temperature silicon carbide power devices.

Jean-Pierre Chante was born in Lyon, France, in 1942. He received the "Doctorat d'etat' from the University of Lyon, France, in 1981. From 1980 to 1986, he managed a research team in the field of power semicconductor devices at the Ecole Centrale de Lyon.

Since 1986 he has been a Professor of Electronic Components and Applied Electronics at the INSA, where he is the head of the Power Devices and Applications team which is part of the CEGELY. He is also in charge of the Centre Inter-universitaire de Microélectronique de la Région de Lyon, which is a regional research center in the microelectronic field.

His interests are in high-temperature electronics, SiC-based components, advanced power devices and CAD tools for power electronics.

Dr. Chante is the author or co-author of numerous papers on these subjects. $\mathrm{He}$ is a member of the SEE (Société des Electriciens et des Electroniciens). 